\title{
Biology and Clinical Significance of Virulence Plasmids in Salmonella Serovars
}

\author{
Donald G. Guiney, Ferric C. Fang, Martin Krause, \\ Stephen Libby, Nancy A. Buchmeier, and Joshua Fierer
}

\begin{abstract}
From the Departments of Medicine and Pathology. School of Medicine, University of California at San Diego, and the Departments of Medicine and Pathology, Veterans Affairs Medical Center, San Diego, California; the Departments of Medicine, Pathology, and Microbiology, University of Colorado Health Sciences Center, Denver, Colorado; and the Department of Medicine, University of Zurich, Zurich, Switzerland
\end{abstract}

\begin{abstract}
Non-typhoid Salmonella strains containing virulence plasmids are highly associated with bacteremia and disseminated infection in humans. These plasmids are found in Salmonella serovars adapted to domestic animals, such as Salmonella dublin and Salmonella choleraesuis, as well as in the widely distributed pathogens Salmonella typhimurium and Salmonella enteritidis. Although virulence plasmids differ between serovars, all contain a highly conserved 8 -kb region containing the $s p v$ locus that encodes the $s p v R$ regulatory gene and four structural $s p v A B C D$ genes. Studies in mice suggest that the spv genes enhance the ability of Salmonella strains to grow within cells of the reticuloendothelial system. The spv genes are not expressed during exponential growth in vitro but are rapidly induced following entry of Salmonella strains into mammalian cells, including macrophages. Transcription of the spv genes is controlled by the stationary-phase $\sigma$ factor RpoS, and mutations in RpoS abolish virulence. These studies suggest that the ability of Salmonella strains to respond to starvation stress in the host tissues is an essential component of virulence.
\end{abstract}

Organisms of the genus Salmonella cause three distinct clinical syndromes: (1) gastroenteritis, (2) enteric (typhoid) fever, and (3) septicemia, with frequent metastatic foci of infection. The most common manifestation of salmonella infection is self-limited enteritis, and dissemination of bacteria outside of the gastrointestinal tract is unusual in the healthy host. In contrast, systemic disease is the hallmark of enteric fever, with bacteremia and infection of lymphoid tissue and the reticuloendothelial system. Clinical gastroenteritis is not prominent, but late complications of Peyer's patch infection, which involve perforation and hemorrhage, are particularly distinctive features of enteric fever and are uncommon in other salmonella infections. Disseminated disease is also seen in the third form of salmonellosis, but in this syndrome, severe sepsis may dominate the clinical picture or patients may present with localized foci of infection; gastrointestinal symptoms may be mild or absent, and the bowel complications seen in enteric fever are not present [1].

Although a very large number of distinct Salmonella strains have been identified by a combination of serological, biological, and genetic techniques, a limited number of strains have been shown to be closely associated with either the enteric

Grant support: This work was supported by the National Institutes of Health (AI32.178 to D.G.G., DK35108 to D.G.G. and J.F., AI32463 to F.C.F.); the United States Department of Agriculture (93-37204 to D.G.G. and 94-01954 to F.C.F.); and the Swiss National Science Foundation (32-32401.91 and 32039342.93 to M.K.)

Reprints or correspondence: Dr. Donald G. Guiney, Department of Medicine, School of Medicine, University of California San Diego, La Jolla, California 92093-0640.

Clinical Infectious Diseases 1995; 21(Suppl 2):S146-51 1995 by The University of Chicago. All rights reserved. $1058-4838 / 95 / 2104-0002 \$ 02.00$ fever or septicemia syndromes. Only Salmonella typhi and, occasionally, Salmonella paratyphi serovars have been shown to cause typhoid fever. These strains are host adapted to humans and do not cause disease in animals. Both Salmonella choleraesuis and Salmonella dublin are strongly associated with the septicemic/metastatic form of salmonellosis, while these organisms are uncommon causes of gastroenteritis in humans [1, 2]. In contrast, $>2,000$ different serovars have been identified among isolates from patients with gastroenteritis, and a few of these, notably Salmonella typhimurium and Salmonella enteritidis, are also prominent isolates in cases of salmonella bacteremia [2].

These observations indicate that Salmonella strains differ in their ability to produce particular disease syndromes. The molecular genetic basis for the different virulence traits among these strains remains poorly understood. However, recent evidence indicates that a common plasmid-encoded genetic locus is a major determinant of virulence in non-typhoid Salmonella serovars associated with systemic disease [3, 4]. Virulence plasmids in Salmonella strains were identified by several early studies in which large "cryptic" plasmids were cured, and the plasmid-free derivatives were found to be less virulent in mice. Subsequent studies in which virulence was restored by reintroduction of the plasmids provided definitive evidence for plasmid-encoded virulence genes $[3,4]$.

\section{Distribution of Virulence Plasmids in Salmonella Serovars}

Virulence plasmids are found in only a small number of Salmonella serovars, and these can be divided into two groups based on their epidemiology. One group consists of serovars that are host adapted to domestic animals: $S$. dublin (cattle), 
S. choleraesuis (pigs), Salmonella gallinarum-pullorum (fowl), and Salmonella abortusovis (sheep) [5, 6]. The host adaptation is reflected by the observations that the particular serovar is frequently associated with disseminated disease in its host species but is usually not found in other animal species. S. dublin and $S$. choleraesuis are also associated with systemic disease in humans, while $S$. gallinarum-pullorum and $S$. abortusovis are not human pathogens. It is significant that virulence plasmids have not been found in $S$. typhi, despite its properties of human adaptation and propensity to cause systemic illness [5]. The second group of Salmonella strains carrying virulence plasmids are the broad host-range serovars $S$. typhimurium and $S$. enteritidis [5]. These organisms are isolated from a variety of hosts and are prominent causes of both the gastroenteritis and septicemia syndromes in humans.

Virulence plasmids range in size from $50 \mathrm{~kb}$ to $100 \mathrm{~kb}$, and considerable differences exist between plasmids from individual serovars. However, plasmids isolated from strains of a given serovar are remarkably similar. These observations suggest that the plasmids are specific to the particular serovar and that little if any plasmid exchange occurs between serovars. These findings are consistent with the apparent absence of complete self-transmissible conjugation systems on the virulence plasmids [7]. Heteroduplex analysis indicates that the larger $S$. typhimurium plasmid $(100 \mathrm{~kb})$ contains a region hybridizing to virtually all of the $S$. enteritidis plasmid $(60 \mathrm{~kb})$ and almost all of the $S$. choleraesuis plasmid $(50 \mathrm{~kb})$. In contrast, large regions of the $S$. dublin plasmid $(80 \mathrm{~kb})$ appear unrelated to any of the sequences of the other virulence plasmids tested [8]. Despite these differences, the plasmid from $S$. enteritidis was found to restore mouse virulence to a plasmid-cured $S$. dublin strain [9]. In a similar vein, the plasmid from $S$. gallinarumpullorum is able to substitute for the $S$. typhimurium plasmid in mouse virulence, despite the fact that $S$. gallinarum-pullorum strains are not virulent in mice [10]. The results indicate that virulence plasmids are not responsible for the host-adaptation phenotype, but rather encode a common virulence function that is interchangeable among the host serovars. Subsequent studies have identified a highly conserved region of $\sim 8 \mathrm{~kb}$, designated $s p v$ for Salmonella plasmid virulence, present on all the plasmids and responsible for the virulence phenotype in mice. The $s p v$ locus consists of the $s p v R$ regulatory gene and four structural genes, $\operatorname{sp} A B C D$.

\section{Contribution of the $s p v$ Locus to the Virulence of Salmonella Serovars}

Most of the work on the virulence plasmid phenotype has been done with inbred mice homozygous for the Ity ${ }^{\text {s locus. }}$ These mice are much more susceptible to Salmonella strains than are animals carrying the $\mathrm{Ity}^{\mathrm{R}}$ allele. In Ity ${ }^{\mathrm{S}}$ mice, $S$. typhimurium, $S$. dublin, $S$. enteritidis, and $S$. choleraesius strains cured of their respective plasmids are $10^{1}-$ to $10^{6}$-fold less virulent than their isogenic wild-type parent strains $[3,4]$. This loss of virulence is generally seen whether the inoculum is given intraperitoneally or orally, although certain S. typhimurium strains may show a plasmid effect only with oral infection [11]. The plasmid virulence phenotype is also expressed in Ity ${ }^{R}$ mice. Detailed studies on the pathogenesis and histopathology of oral salmonella infection in mice show that the plasmid is not required for invasion of the bacteria into intestinal epithelial cells and Peyer's patches, nor for spread to mesenteric nodes and the reticuloendothelial system (RES) of the liver and spleen. Instead, the plasmid-containing strains outgrow plasmid-free bacteria in the RES, leading to an overwhelming infection characterized by prominent microabscess formation and large numbers of organisms in the liver and spleen prior to death at 6-8 days after inoculation [12]. The plasmid-free strain of $S$. dublin was found to invade the bowel wall and ileal Peyer's patches with an efficiency equal to that of the wild-type parent strain. However, plasmid-free bacteria in the mesenteric lymph nodes and the spleen increased much more slowly than wild-type, remaining $10^{2}$ - to $10^{3}$-fold lower in number at 7 days after infection. After 10 days, the number of plasmid-free bacteria decreased and the liver histology showed numerous small granulomas. These results indicate that the slower growth of plasmid-free strains allows the host to mount an effective immune response. Polymorphonuclear leukocytes do not appear to be important in controlling the initial growth of plasmid-free strains, since the numbers of bacteria recovered from neutropenic mice over the first 7 days of infection were the same as those for control mice [12].

Several lines of evidence suggest that the virulence plasmid enhances bacterial growth within the intracellular environment of host cells. In the murine model, Salmonella strains rapidly enter cells following inoculation [13]. Gentamicin treatment of mice infected with plasmid-containing $S$. dublin does not alter the lethal course of infection, owing to the inability of the antibiotic to enter host cells. However, mice are cured of salmonella infection by the administration of gentamicin incorporated into liposomes, which enables delivery of the drug to the intracellular environment [14]. Results of studies in which a temperature-sensitive marker plasmid is used in $S$. typhimurium suggest that the virulence plasmid accelerates intracellular growth of bacteria in the liver and spleen [15], and recent evidence indicates that the vast majority of viable plasmid-containing bacteria in the spleen are found in the macrophages within 3 days after infection (N. A. Buchmeier, unpublished observations). Plasmid-containing strains do not appear to cause any general immunosuppression, since the growth of plasmid-free strains is not enhanced by mixed infection [16]. Results of these in vivo studies suggest that the virulence plasmid increases bacterial growth within macrophages of the RES, although growth within other host cells may occur as well.

Only limited studies of the virulence plasmid phenotype have been done in humans and domestic animal hosts. The virulence plasmid is required for $S$. gallinarum-pullorum to produce severe systemic disease in fowl [17]. The $S$. choleraesuis plasmid 
also appears to enhance disease in pigs [18]. Since only a portion of $S$. typhimurium strains carry a virulence plasmid, it is possible to use molecular epidemiology to determine whether plasmid-containing strains are more closely associated with systemic disease than with gastroenteritis. By using this approach, we have found that $76 \%$ of strains isolated from blood cultures but only $42 \%$ of unrelated fecal isolates carried a virulence plasmid; these findings provide evidence that the virulence plasmid plays a significant role in human disease [19]. Similar results have also been reported in studies of domestic livestock [8]. These findings confirm the general significance of virulence plasmids in systemic disease due to nontyphoid Salmonella serovars.

The absence of virulence plasmid sequences in S. typhi and the $S$. paratyphi serovars associated with enteric fever suggests that the pathogenesis of typhoid is quite different from that of disseminated, non-typhoid salmonellosis. Another difference is the presence of the $\mathrm{Vi}$ capsule in $S$. typhi and its absence in most virulence plasmid-containing isolates. $S$. typhi is not pathogenic for mice, and introduction of a virulence plasmid does not restore virulence in murine infections [5]. This result indicates that crucial differences in chromosomal genes exist between $S$. typhi and the mouse virulent serovars. Chromosomal loci required for mouse virulence are also missing from a number of non-typhoid serovars. Transfer of a virulence plasmid to the Salmonella serovars $S$. derby, $S$. havana, $S$. minnesota, S. ohio, and S. saintpaul failed to confer mouse virulence on these strains, while $S$. heidelberg and $S$. newport were rendered virulent [5]. These studies indicate that both the virulence plasmid and the chromosome are key determinants of mouse virulence. Since $S$. typhi differs in both of these elements, salmonellosis in mice has severe limitations as a model for typhoid fever and more likely parallels the septicemia syndrome.

\section{Molecular Analysis of the $\boldsymbol{S}$. dublin Plasmid pSDL2}

In early studies in which insertion mutations and deletions were used, a single virulence region on the $S$. dublin plasmid was identified, as well as a fragment capable of autonomous replication (figure 1) [9]. Subsequent experiments showed that an 8.2-kb segment from the virulence region cloned on an IncP plasmid replicon could restore virulence to a plasmid-cured S. dublin strain. Complete sequence analysis of this fragment revealed six open reading frames, currently designated spvRABCD and orfE (figure 1) [20]. Comparison with spv sequences from S. typhimurium and S. choleraesuis show $<0.5 \%$ divergence at the nucleotide level [3]. An insertion sequencelike element related to IS630 is located upstream from $s p v R$ [21]. Early work identified a region downstream from orfE required for plasmid stability [9]. This region was found to contain a resolvase $(r s d)$ closely related to the $\mathrm{D}$ protein of miniF and a cis-acting resolution site, designated crs [22]. The crs element contains eight direct, incomplete 17 -bp repeats and

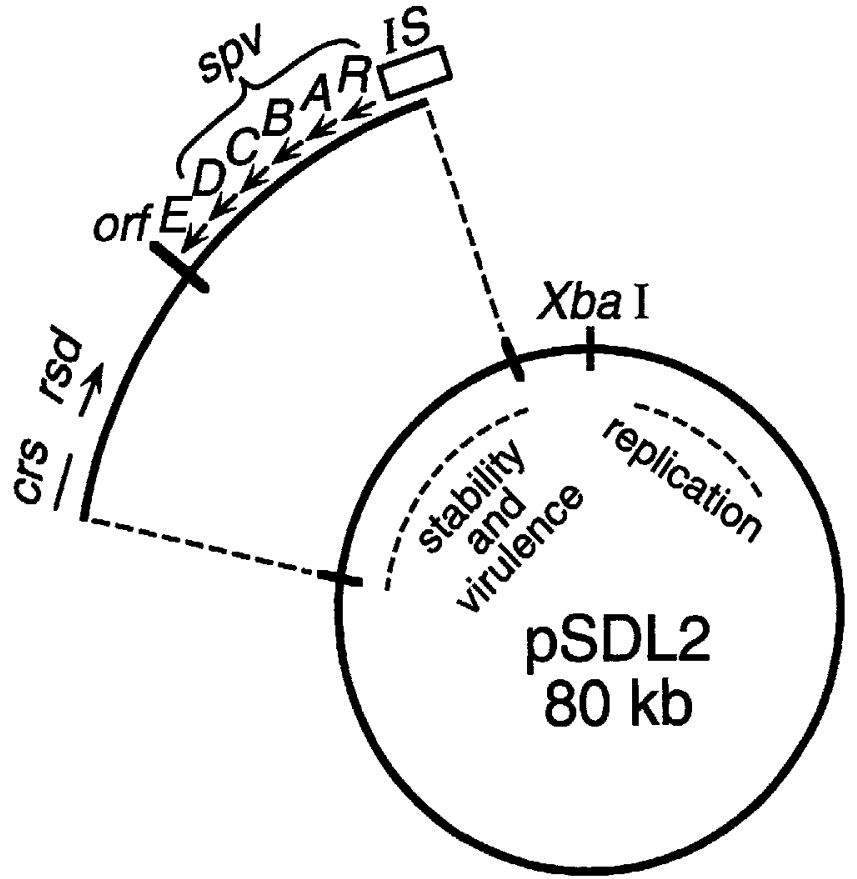

Figure 1. Map of known genetic loci on the Salmonella dublin virulence plasmid pSDL2. A region encoding autonomous replication functions is located clockwise from the single $X b a$ I site. The essential virulence locus consists of the five $s p v R A B C D$ genes, bordered by a 1S630-like element upstream and orf $E$ downstream. A region affecting plasmid stability constitutes a multimer resolution system, composed of a gene for resolvase $(r s d)$ and a crossover site for specific recombination (crs), leading to the conversion of plasmid multimers to monomers.

a region of indirect repeats homologous to oriVI of $F$. The $r s d / c r s$ system is capable of resolving multimers generated in $\mathrm{recA}^{+}$hosts and of stabilizing heterologous replicons in Salmonella strains. A resolvase function active on crs was found in other Salmonella serovars harboring a virulence plasmid, suggesting that this system is a general mechanism for virulence plasmid stabilization.

Deletion and insertion mutagenesis has been used to define the plasmid genes essential for virulence in S. dublin. Tn5-oriT insertions in orfE retain virulence, but deletion of $s p v D$ partially attenuates virulence [20]. Tn.5-oriT inserts in $s p v R, s p v B$, and $s p v C$ abolish virulence [20]. Due to the polar nature of $\operatorname{Tn} 5$ inserts, non-polar mutations using an $\mathrm{Xba}$ I linker oligonucleotide were also constructed [23]. Analysis of these plasmids showed that $s p v A$ is not essential for virulence in mice. Mutations throughout $s p v B$ are nonvirulent, including stop-codon inserts close to the $\mathrm{COOH}$-terminus. Mutations in $s p v C$ and $s p v D$ are partially virulent but the plasmids are unstable in vivo. Insertions in $s p v R$ are avirulent and abolish production of the SpvABCD proteins, consistent with the essential regulatory role of $s p v R$ (see below). Taken together, the results indicate that $s p v R$ and $s p v B$ are essential virulence genes in mice and that $s p v C$ and $s p v D$ have accessory roles and are probably needed for full virulence, while $s p v A$ is not essential. In 
S. typhimurium, $s p v C$ and $s p v D$ have significant roles in mouse virulence $[24,25]$.

The $s p v$ genes are transcribed from two promoter regions, one located upstream from $s p v R$ and the second positioned between $s p v R$ and $s p v A$ [26]. Transcription of the $s p v A B C D$ genes is initiated upstream from $s p v A$, and messages for $s p v A$, $s p v A B$, $s p v A B C$, and $s p v A B C D$ are found in decreasing abundance. LacZ translational fusions to the Spv proteins are also expressed with the same decreasing activity, SpvA being highest and SpvD lowest [26]. These findings suggest that transcripts initiate at the $s p v A$ promoter and can terminate within each of the long intergenic regions. However, a complex system of termination and mRNA processing cannot be excluded. The promoter for $s p v R$ has not been localized, but a monocistronic message is found, indicating that $s p v R$ transcription normally terminates before the $s p v A$ gene (M. Krause and D. G. Guiney, unpublished observations).

\section{Regulation of spv Expression}

The discovery that $s p v$ expression varies during the bacterial growth phase provided the key to understanding the mechanism of $s p v$ gene regulation shown in figure 2 [27]. When LacZ translational fusions were used, the expression of $s p v A B C D$ was found to be very low in early log-phase cells and to increase rapidly in the post-exponential phase [26, 27]. This growth-phase regulation was confirmed by analysis of $s p v$ mRNA transcribed in wild-type S. dublin [26]. In the log phase, only small quantities of single-length $s p v A$ mRNA are made. As cells enter the stationary phase, progressively longer messages are found, including full-length $s p v A B C D$ mRNA.

Sequence analysis of SpvR suggested that this protein belongs to the large family of LysR/MetR-like transcriptional activators [28]. These proteins act as single-component regulators of gene expression, and they contain a conserved helixturn-helix motif in the $\mathrm{NH}_{2}$-terminal region involved in DNA interaction [29]. Genetic studies confirmed that SpvR is absolutely required for $s p v A B C D$ expression [27]. Knockout mutations in $s p v R$ abolish the synthesis of LacZ fusions with the structural Spv proteins, as well as production of the native $\mathrm{Spv}$ proteins in minicells $[23,26,27]$. SpvR binds to a region upstream from the spvA transcriptional start sites, and mutations in the helix-turn-helix motif abolish binding in vitro and activation of $\operatorname{spv} A$ transcription in vivo [30]. However, overproduction of SpvR from constitutive promoters does not abolish the growth-phase regulation of the $s p v A B C D$ structural genes, indicating that the SpvR level is not the sole regulator of $s p v$ expression [26].

The pattern of $s p v$ growth-phase regulation strongly suggested the involvement of the alternative $\sigma$ factor RpoS (KatF, $\left.\sigma^{s}\right)$. The activity of this $\sigma$ factor is induced during post-exponential growth and regulates the stationary-phase expression of a large set of target genes involved in starvation survival and environmental stress responses [31]. The central role of RpoS

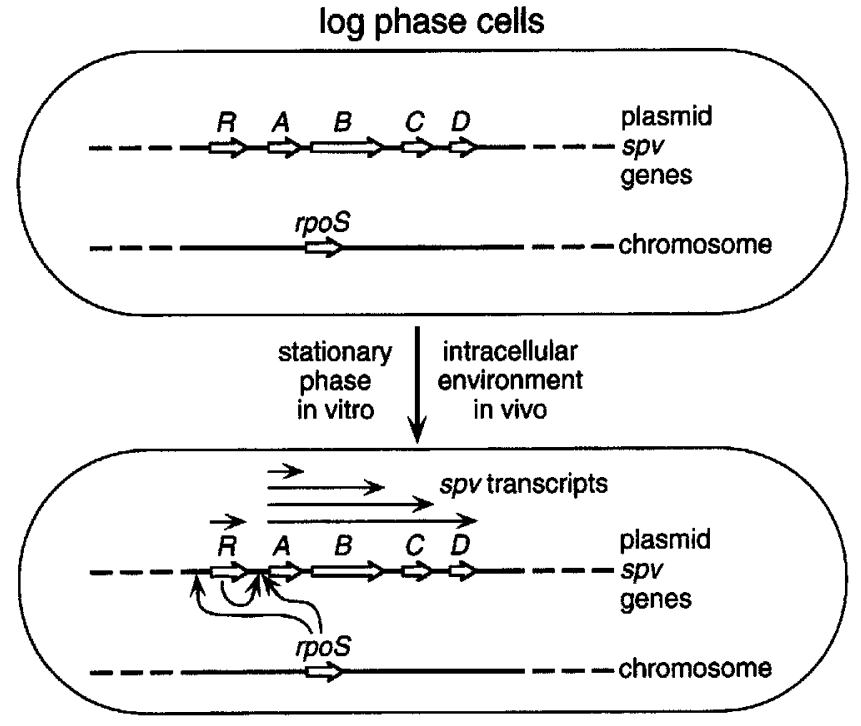

Figure 2. Mechanism of $s p v$ gene regulation on the virulence plasmids of Salmonella serovars. Bacterial cells growing in logarithmic phase in rich culture media do not express the $s p v$ genes (depicted in the upper cell). However, both post-exponential growth in vitro and the intracellular environment of host cells in vivo induce the $s p v$ genes (shown in the lower cell). Induction depends on both the chromosomal $\sigma$ factor RpoS and the plasmid regulatory protein SpvR. RpoS activity increases in stationary phase and in response to nutrient starvation. RpoS appears to act at both the $s p v R$ and $s p v A$ promoters. SpvR is essential for transcription of the $s p v A B C D$ genes by binding to the $s p v A$ promoter region. Transcription of the $s p v A B C D$ structural genes is initiated at the $s p v A$ promoter, and multiple transcripts are found.

in $s p v$ gene expression was demonstrated by constructing an rpoS knockout mutation in $S$. typhimurium [32]. Expression of $s p v B$ is severely decreased in the rpoS mutant. As expected, the rpoS mutant is markedly attenuated for virulence in mice. Recent evidence indicates that RpoS is required for optimal transcription at both the $s p v R$ and $s p v A$ promoters (figure 2) ([33] and D. G. Guiney, unpublished observations).

\section{Induction of $s p v$ Gene Expression by the Host-Cell Environment}

Since considerable indirect evidence suggests that the $s p v$ genes are active in the intracellular environment of the host, the expression of the $s p v$ genes was examined after uptake of bacteria by mammalian cells in tissue culture [34]. Fluorescent (and later luminescent) substrates of LacZ were used to develop a sensitive assay for the expression of $s p v:: l a c Z$ fusions by small numbers of bacteria inside eukaryotic cells. These studies have shown that the $s p v$ genes are rapidly induced by $>100$ fold on uptake by macrophages, epithelial cells, and hepatocytes ([34] and S. Libby, J. Fierer, and D. G. Guiney, unpublished data). Most of the induction occurs within the first hour following uptake and does not depend on acidification of the 
intracellular vacuole containing the Salmonella strain. This regulation differs considerably from control of the chromosomal locus pagC; pagC is regulated by the PhoP/PhoQ two-component system and is induced in macrophages, but not epithelial cells, by a mechanism requiring phagosome acidification over a period of 3 hours [35]

These results indicate that $s p v$ induction occurs in response to a general property of the intracellular environment, perhaps reflecting a relative lack of nutrients available within the endocytic or phagocytic vacuole. Since the intracellular induction of the $s p v$ genes requires both SpvR and RpoS, the genetic mechanisms regulating post-exponential phase synthesis in vitro are the same as those controlling the $s p v$ operon after entry into host cells, and presumably throughout the infectious process (figure 2). The finding that virulence genes are regulated by RpoS suggests that Salmonella strains experience significant starvation stress during intracellular infection. Since the RpoS-mediated response increases bacterial resistance to a variety of adverse environmental factors, this regulation appears to be essential for the organism to survive and multiply in the host.

\section{Plasmid-Cured Salmonella Strains as Live Vaccines}

Since plasmid-cured $S$. dublin produces a self-limited systemic infection with granuloma formation, the ability of this strain to induce protective immunity was tested in mice [36]. Animals immunized by either oral or intraperitoneal inoculation were protected against lethal challenge with virulent $S$. dublin given by oral or intraperitoneal routes of infection. The fact that intraperitoneal vaccination could protect against a virulent oral challenge led to the demonstration that systemic as well as oral salmonella vaccination produces infection of Peyer's patches in the intestine. Mice immunized with $S$. dublin (group D) were protected against challenge with other group D Salmonella serovars and also against $S$. typhimurium (group B), which shares an O-antigen determinant with group D [36]. Similar results were reported with S. enteritidis (group D) [37]. However, $S$. dublin does not protect mice from $S$. choleraesuis (group $\mathrm{C}$ ), which lacks any cross-reactive $\mathrm{O}$-antigen epitopes [36]. It is likely that both lipopolysaccharide and non-lipopolysaccharide antigens are important in the protection induced by live, plasmid-cured strains. These immunization studies have important implications for vaccine development. The use of the plasmid-free strain as a vaccine shows that an immune response to the $\mathrm{Spv}$ proteins is not required for protection. Since plasmid-containing Salmonella serovars are major pathogens of domestic animals, plasmid-free derivatives could be combined in a multivalent vaccine for use in livestock.

\section{Conclusions}

The plasmid-encoded $s p v$ genes enhance the ability of certain non-typhoid Salmonella serovars to produce severe, extraintes- tinal disease. The $s p v$ locus consists of the $s p v R$ regulatory gene and the four structural $s p v A B C D$ genes. Studies on $s p v$ gene regulation have established the importance of post-exponential or stationary-growth-phase control mechanisms on the expression of virulence genes in Salmonella strains. The alternative $\sigma$ factor RpoS, together with SpvR, regulates expression of the $s p v$ operon and ensures rapid induction of the $s p v$ genes within the intracellular environment of the host. Evidence from pathogenesis studies in mice suggests that the $s p v$ genes enhance the growth of Salmonella species within cells of the RES.

In clinical practice, non-typhoid salmonella bacteremia and metastatic infection are associated with a variety of predisposing conditions that decrease natural or acquired immunity, including extremes of age, decreased gastric acidity, malignancy, immunosuppression, and AIDS. The Salmonella serovars causing systemic disease in these patients are predominantly strains that carry a virulence plasmid. The virulence-plasmid phenotype does not appear to interfere with clearance by acquired immune mechanisms. By extrapolation from the mouse model, the $s p v$ genes are likely to facilitate growth of the Salmonella strains within host cells at the sites of systemic infection, particularly in patients who are unable to mount an effective immune response. Salmonella infection in patients receiving immunosuppressive therapy or in patients with AIDS is particularly difficult to eradicate. The identification of the $s p v$ locus provides a molecular genetic explanation for the propensity of plasmid-containing serovars to cause serious infections in these patients.

\section{References}

1. Fang FC, Fierer J. Human infection with Salmonella dublin. Medicine (Baltimore) 1991; 70:198-207.

2. Blaser MJ, Feldman RA. Salmonella bacteremia: reports to the Centers for Disease Control, 1968-1979. J Infect Dis 1981;143:743-6.

3. Gulig PA, Danbara H, Guiney DG, Lax AJ, Nore F, Rhen M. Molecular analysis of $s p v$ virulence genes of the salmonella virulence plasmids. Mol Microbiol 1993; 7:825-30.

4. Guiney DG, Fang FC, Krause M, Libby S. Plasmid-mediated virulence genes in non-typhoid Salmonella serovars. FEMS Microbiol Lett 1994; $124: 1-10$.

5. Roudier C, Krause M, Fierer J, Guiney DG. Correlation between the presence of sequences homologous to the vir region of Salmonella dublin plasmid pSDL2 and the virulence of twenty-two Salmonella serotypes in mice. Infect Immun 1990;58:1180-5.

6. Colombo MM, Leori G, Rubino S, Barbato A, Cappuccinelli P. Phenotypic features and molecular characterization of plasmids in Salmonella abortusovis. J Gen Microbiol 1992; 138:725-31.

7. Chikami GK, Fierer J, Guiney DG. Plasmid-mediated virulence in Salmonella dublin demonstrated by use of a Tn5-oriT construct. Infect Immun $1985 ; 50: 420-4$.

8. Montenegro MA, Morelli G, Helmuth R. Heteroduplex analysis of Salmonella virulence plasmids and their prevalence in isolates of defined sources. Microb Pathog 1991;11:391-7.

9. Beninger PR, Chikami G, Tanabe K, Roudier C, Fierer J, Guiney DG. Physical and genetic mapping of the Salmonella dublin vinulence plas- 
mid pSDL2. Relationship to plasmids from other Salmonella strains. J Clin Invest 1988;81:1341-7.

10. Barrow PA, Lovell MA. Functional homology of virulence plasmids in Salmonella gallinarum, S. pullorum, and $S$. typhimurium. Infect Immun 1989;57:3136-41.

11. Gulig PA, Curtiss R III. Plasmid-associated virulence of Salmonella typhimurium. Infect Immun 1987;55:2891-901.

12. Heffernan EJ, Fierer J, Chikami G, Guiney D. Natural history of oral Salmonella dublin infection in BALB/c mice: effect of an 80-kilobasepair plasmid on vinulence. $J$ Infect Dis 1987; 155:1254-9.

13. Dunlap NE, Benjamin WH Jr, McCall RD Jr, Tilden AB, Briles DE. A "safe-site" for Salmonella typhimurium is within splenic cells during the early phase of infection in mice. Microb Pathog 1991; 10:297-310.

14. Fierer J, Hatlen L, Lin JP, Estrella D, Mihalko P, Yau-Young A. Successful treatment using gentamicin liposomes of Salmonella dublin infections in mice. Antimicrob Agents Chemother 1990;34:343-8.

15. Gulig PA, Doyle TJ. The Salmonella typhimurium vinulence plasmid increases the growth rate of salmonellae in mice. Infect Immun 1993;61:504-11.

16. Gulig PA, Curtiss R III. Cloning and transposon insertion mutagenesis of virulence genes of the 100-kilobase plasmid of Salmonella typhimurium. Infect Immun 1988;56:3262-71.

17. Barrow PA, Simpson JM, Lovell MA, Binns MM. Contribution of Salmonella gallinarum large plasmid toward virulence in fowl typhoid. Infect Immun 1987;55:388-92.

18. Danbara $H$, Moriguchi $R$, Suzuki $S$, et al. Effect of 50-kilobase plasmid pKDSC50 of Salmonella choleraesuis RF-1 strain on pig septicemia. J Vet Med Sci 1992;54:1175-8.

19. Fierer J, Krause M, Tauxe R, Guiney D. Salmonella typhimurium bacteremia: association with the virulence plasmid. J Infect Dis 1992; 166:639-42.

20. Krause M, Roudier C, Fierer J, Harwood J, Guiney D. Molecular analysis of the virulence locus of the Salmonella dublin plasmid pSDL2. Mol Microbiol 1991;5:307-16.

21. Krause M, Harwood J, Fierer J, Guiney D. Genetic analysis of homology between the virulence plasmids of Salmonella dublin and Yersinia pseudotuberculosis. Infect Immun 1991;59:1860-3.

22. Krause $M$, Guiney DG. Identification of a multimer resolution system involved in stabilization of the Salmonella dublin virulence plasmid pSDL2. J Bacteriol 1991; 173:5754-62.

23. Roudier C, Fierer J, Guiney DG. Characterization of translation termination mutations in the $s p v$ operon of the Salmonella virulence plasmid pSDL2. J Bacteriol 1992; 174:6418-23.
24. Gulig PA, Chiodo VA. Genetic and DNA sequence analysis of the Salmonella typhimurium virulence plasmid gene encoding the 28000 molecular weight protein. Infect Immun 1990;58:2651-8.

25. Gulig PA, Caldwell AL, Chiodo VA. Identification, genetic analysis, and DNA sequence of a 7.8-kb virulence region of the Salmonella typhimurium virulence plasmid. Mol Microbiol 1992;6:1395-411.

26. Krause M, Fang FC, Guiney DG. Regulation of plasmid virulence gene expression in Salmonella dublin involves an unusual operon structure. J Bacteriol 1992; 174:4482-9.

27. Fang FC, Krause M, Roudier C, Fierer J, Guiney DG. Growth regulation of a Salmonella plasmid gene essential for virulence. J Bacteriol 1991; 173:6783-9.

28. Pullinger GD, Baird GD, Williamson CM, Lax AJ. Nucleotide sequence of a plasmid gene involved in the virulence of salmonellas. Nucl Acid Res 1989; 17:7983.

29. Henikoff S, Haughn GW, Calvo JM, Wallace JC. A large family of bacterial activator proteins. Proc Natl Acad Sci USA 1988;85:6602-6

30. Krause M, Fang FC, El-Gedaily A, Libby S, Guiney DG. Mutational analysis of SpvR-binding to DNA in the regulation of the Salmonella plasmid virulence operon. Plasmid 1995 (in press).

31. Loewen PC, Hengge-Aronis R. The role of the sigma factor $\sigma^{s}$ (KatF) in bacterial global regulation. Annu Rev Microbiol 1994;48:53-80.

32. Fang FC, Libby SJ, Buchmeier NA, Loewen PC, Switala J, Harwood J, Guiney DG. The alternative $\sigma$ factor KatF (RpoS) regulates Salmonella virulence. Proc Natl Acad Sci USA 1992;89:11978-82.

33. Kowarz L, Coynault C, Robbe-Saule V, Norel F. The Salmonella typhimurium kat $F$ (rpoS) gene: Cloning, nucleotide sequence, and regulation of $s p v R$ and $s p v A B C D$ virulence plasmid genes. J Bacteriol 1994; 176:6852-60.

34. Fierer J, Eckmann L, Fang F, Pfeifer C, Finlay BB, Guiney D. Expression of the Salmonella virulence plasmid gene $s p v B$ in cultured macrophages and nonphagocytic cells. Infect Immun 1993;61:5231-6.

35. Alpuche Aranda CM, Swanson JA, Loomis WP, Miller SI. Salmonella typhimurium activates virulence gene transcription within acidified macrophage phagosomes. Proc Natl Acad Sci USA 1992;89:10079-83.

36. Fierer J, Chikami G, Hatlen L, Heffernan EJ, Guiney D. Active immunization with LD842, a plasmid-cured strain of Salmonella dublin, protects mice against group D and group B Salmonella infection. J Infect Dis $1988 ; 158: 460-3$.

37. Nakamura M, Sato S, Ohya T, Suzuki S, Ikeda S, Koeda T. Plasmid-cured Salmonella enteritidis AL 1192 as a candidate for a live vaccine. Infect Immun 1985; 50:586-7. 